\title{
SOME APPLICATIONS OF AN INEQUALITY IN LOCALLY CONVEX SPACES
}

\author{
BY \\ C. W. McARTHUR( $\left.{ }^{(}\right)$AND J. R. RETHERFORD $\left({ }^{2}\right)$
}

1. Introduction. Throughout this paper $(X, \tau)$ will denote a locally convex Hausdorff linear topological space with additional hypotheses added as needed. Our aim is to present some rather diverse applications of a fundamental inequality valid in any vector space and denoted by (I) below.

In $\S 4$ inequality (I) is used to give a simple proof of the uniform convergence of an unconditionally convergent series with respect to various classes of bounded multipliers. The theorem obtained includes recent results of Veic [10] and Weill [11].

In $\S 5$ we study some properties of series having unordered bounded partial sums, continuing the study of [6]. In $\$ 7$ we consider some compact linear transformations determined by series, and in $\S 8$ we obtain a stability theorem for Schauder bases in Banach spaces and discuss its relationship with a stability theorem of Veic [10] and a classical stability theorem of Krein, Milman and Rutman [5].

2. The inequality. Let $\sigma$ denote a finite set of positive integers, $\left(x_{j}\right)_{j \in \sigma}$ a family of elements of $X$ and $\left(t_{j}\right)_{j \in \sigma}$ a family of scalars. For each seminorm $\rho$ on $X$ the following inequality is valid:

$$
\rho\left(\sum_{j \in \sigma} t_{j} x_{j}\right) \leqq 4 \sup _{j \in \sigma}\left|t_{j}\right| \sup _{\sigma^{\prime} \subset \sigma} \rho\left(\sum_{j \in \sigma^{\prime}} x_{j}\right) .
$$

To see that (I) is valid first assume all scalars are nonnegative real numbers with $t_{1} \geqq t_{2} \geqq \cdots \geqq t_{n}$. Then

$$
\begin{aligned}
\rho\left(\sum_{j=1}^{n} t_{j} x_{j}\right) & =\rho\left[\sum_{j=1}^{n-1}\left(t_{j}-t_{j+1}\right)\left(x_{1}+\cdots+x_{j}\right)+t_{n}\left(x_{1}+\cdots+x_{n}\right)\right] \\
& \leqq \sum_{j=1}^{n-1}\left(t_{j}-t_{j+1}\right) \rho\left(x_{1}+\cdots+x_{j}\right)+t_{n} \rho\left(x_{1}+\cdots+x_{n}\right) \\
& \leqq\left[\sum_{j=1}^{n-1}\left(t_{j}-t_{j+1}\right)+t_{n}\right] \sup _{\sigma^{\prime} \subset \sigma} \rho\left(\sum_{j \in \sigma^{\prime}} x_{j}\right) \\
& =\sup _{j \in \sigma}\left|t_{j}\right| \sup _{\sigma^{\prime} \subset \sigma} \rho\left(\sum_{j \in \sigma^{\prime}} x_{j}\right) .
\end{aligned}
$$

Now for real scalars, apply this to the positive and negative scalars separately. Then

Received by the editors September 9, 1966 and, in revised form, December 20, 1967.

(1) Research supported by NSF GP 5952.

( $\left.{ }^{2}\right)$ Research supported by NSF GP 5854. 
for $t_{j}=a_{j}+i b_{j}$, write $\sum t_{j} x_{j}$ as $\sum a_{j} x_{j}+i \sum b_{j} x_{j}$ and apply the inequality for real scalars to each sum separately to obtain (I).

Clearly, if the scalar field is real instead of complex we may replace 4 with 2 .

3. A familiar theorem on complex series. Let $\Phi$ denote the collection of all finite subsets of the positive integers.

THEOREM. If $\sum_{i=1}^{\infty} z_{i}$ is a series of complex numbers with the property that

$$
\sup \left\{\left|\sum_{i \in \sigma} z_{i}\right|: \sigma \in \Phi\right\}=K<+\infty
$$

then $\sum_{i=1}^{\infty}\left|z_{i}\right| \leqq 4 K$.

Proof. Put $\rho(z)=|z|$ and let $t_{i}$ be a complex number of modulus 1 and such that $\left|z_{i}\right|=t_{i} z_{i}$. Now apply (I).

4. Some characterizations of unconditional convergence. In this section we assume that $(X, \tau)$ is sequentially complete. Recall that a series $\sum_{i=1}^{\infty} x_{i}$ in $X$ is unconditionally convergent if for each permutation $p(i)$ of the positive integers, $\sum_{i=1}^{\infty} x_{p(i)}$ converges.

Let $(m)$ denote the Banach space of bounded sequences of scalars $b=\left(b_{i}\right)$ with $\|b\|=\sup _{i}\left|b_{i}\right|$.

THEOREM. For a series $\sum_{i=1}^{\infty} x_{i}$ in $X$ the following are equivalent:

(a) $\sum_{i=1}^{\infty} b_{i} x_{i}$ converges for all $b=\left(b_{i}\right) \in(m)$;

(b) $\sum_{i=1}^{\infty} b_{i} x_{i}$ converges for all $b=\left(b_{i}\right)$ with $b_{i}$ either 0 or 1 for each $i$;

(c) $\sum_{i=1}^{\infty} x_{i}$ is unconditionally convergent;

(d) $\sum_{i=1}^{\infty} x_{i}$ is unordered convergent;

(e) $\lim _{n} \sum_{i=1}^{n} b_{i} x_{i}$ exists uniformly for $b=\left(b_{i}\right) \in(m)$ with $\|b\| \leqq 1$;

(f) $\lim _{n} \sum_{i=1}^{n} b_{i} x_{i}$ exists uniformly for $b=\left(b_{i}\right)$ with $b_{i}$ either 0 or 1 for each $i$;

Proof. For the implications (a) $\Leftrightarrow(b) \Leftrightarrow(c) \Leftrightarrow(d)$ see [3, p. 59]. It is obvious that (e) $\Rightarrow$ (f) and (f) $\Rightarrow$ (b). The proof will be completed by showing that (d) $\Rightarrow$ (e). Let $V$ be a closed balanced convex neighborhood of $\theta$. It follows from (d) that there is a positive integer $N$ such that if $\sigma$ is a finite set of positive integers and $\sigma \cap[1, N]$ is void then $4\left(\sum_{i \in \sigma} x_{\imath}\right) \in V$. Let $\rho$ denote the Minkowski functional of $V$ and $b=\left\{b_{i}\right\} \in(m)$ with $\|b\| \leqq 1$. It follows from (I) that if $q>p>N$ then

$$
\rho\left(\sum_{i=p}^{q} b_{i} x_{i}\right) \leqq 4\|b\| \sup _{\sigma \subset[p, q]} \rho\left(\sum_{i \in \sigma} x_{i}\right) \leqq \sup _{\sigma \subset[p, q]} \rho\left(4 \sum_{i \in \sigma} x_{i}\right) \leqq 1
$$

so $\sum_{i=p}^{q} b_{i} x_{i} \in V$ if $q>p>N$ and $\|b\| \leqq 1$.

One should compare the above proof with [10] and [11].

5. Some characterizations of unordered boundedness. In this section $(X, \tau)$ need no longer be sequentially complete. Again let $\Phi$ denote the collection of finite subsets of the positive integers. Also, let $\left(c_{0}\right)$ denote the Banach space of sequences tending to 0 with the sup norm. 
A formal series $\sum_{i=1}^{\infty} x_{i}$ in $(X, \tau)$ is unordered bounded if

$$
\left\{\sum_{i \in \sigma} x_{i}: \sigma \in \Phi\right\} \text { is } \tau \text {-bounded. }
$$

THEOREM. For a formal series $\sum_{i=1}^{\infty} x_{i}$ in $X$ the following are equivalent:

(a) If $B$ is an equicontinuous subset of $X^{*}$ there is a constant $M_{B}$ such that $\sum_{i=1}^{\infty}\left|f\left(x_{i}\right)\right| \leqq M_{B}$ for all $f \in B$;

(b) $\sum_{i=1}^{\infty}\left|f\left(x_{\imath}\right)\right|<+\infty$ for each $f \in X^{*}$;

(c) $\sum_{i=1}^{\infty} x_{i}$ is unordered bounded;

(d) If $\rho$ is an arbitrary continuous seminorm on $X$, then

$$
C_{\rho} \equiv \sup \left\{\rho\left(\sum_{j \in \sigma} x_{j}\right): \sigma \in \Phi\right\}<+\infty
$$

(e) $\sup \left\{\rho\left(\sum_{j \in \sigma} x_{j}: \sigma \in \Phi\right\}<+\infty\right.$ for every $\rho \in \Gamma$, where $\mathrm{I}$ is a family of continuous seminorms which generate the topology $\tau$;

(f) For each $c=\left(c_{i}\right) \in\left(c_{0}\right)$, the partial sums of $\sum_{i=1}^{\infty} c_{i} x_{i}$ form a Cauchy sequence.

Proof. Let $S=\left\{\sum_{i \in \sigma} x_{i}: \sigma \in \Phi\right\}$. Clearly (a) $\Rightarrow(\mathrm{b})$ and (b) implies $S$ is weakly bounded and hence $\tau$-bounded. Thus (b) $\Rightarrow$ (c).

If (c) holds and $\rho$ is a continuous seminorm then clearly $C_{\rho}$ as defined in (d) is finite. That (d) $\Rightarrow(\mathrm{e})$ is obvious. Assume (e) and let $c=\left(c_{i}\right) \in\left(c_{0}\right)$. If $\rho \in \Gamma$ and $\varepsilon>0$ there is a positive integer $N_{\rho}$ such that $i>N_{\rho}$ implies $\left|c_{i}\right|<\varepsilon / 4 C_{\rho}$. Thus if $q>p>N_{\rho}$ where $\rho \in \Gamma$ we obtain from (I)

$$
\rho\left(\sum_{i=p}^{q} c_{i} x_{i}\right) \leqq 4 C_{\rho} \sup _{p \leqq i \leqq q}\left|c_{i}\right|<\varepsilon \quad \text { and (e) } \Rightarrow \text { (f). }
$$

From the well-known fact that $\sum_{i=1}^{\infty} c_{i} a_{i}$ converging for all $\left(c_{i}\right) \in\left(c_{0}\right)$ and fixed scalar sequence $\left(a_{2}\right)$ implies $\sum_{i=1}^{\infty}\left|a_{i}\right|<+\infty$ we see that (f) $\Rightarrow(\mathrm{b})$. Thus we have (b) through (f) equivalent and the implication (a) $\Rightarrow$ (b). Let $B$ be an equicontinuous subset of $X^{*}$ and let $\rho(x)=\sup \{|f(x)|: f \in B\}$. If (b) through (f) hold then by (d) $C_{\rho}=\sup \left\{\rho\left(\sum_{i \in \sigma} x_{i}\right): \sigma \in \Phi\right\}<+\infty$ and thus $\left|f\left(\sum_{i \in \sigma} x_{i}\right)\right| \leqq C_{\rho}$ for all $f \in B$. From the theorem of $\S 3, \sum_{i=1}^{\infty}\left|f\left(x_{i}\right)\right| \leqq 4 C_{\rho}$ for all $f \in B$ and so (a) through (f) are equivalent.

The equivalence of (a), (b), (c) and (f) have been shown [6] by a different method. A version of (d) and (e) is known in Banach spaces to be equivalent to (c). It is interesting to note how our theorem avoids the category proof of [2, Lemma 2 , p. 159].

Historically a series satisfying (f) has been called a w.u.c. series (for weakly unconditionally convergent). The term "weak" in functional analysis now means and will no doubt continue to mean "with respect to the weak topology" and, in view of the theorem, we see that in nonweakly sequentially complete spaces a "w.u.c. series" need not be weakly convergent. Thus feeling that the use of w.u.c. 
is misleading we adopt the symbolism " $\sum_{i=1}^{\infty} x_{i}$ is UB" for a series satisfying any of (a) through (f).

6. UB series which are not u.c. Clearly every unconditionally convergent (u.c.) series is UB and the converse is valid in weakly sequentially complete spaces. It is known (see e.g. [2, p. 159]) that in a Banach space there is a UB series which is not u.c. if and only if $X$ contains a subspace isomorphic to $\left(c_{0}\right)$. In this section we consider the relationship between UB and u.c. series in a sequentially complete locally convex space $(X, \tau)$.

A sequence $\left(x_{i}\right)$ in $X$ is a basic sequence if for each $x \in\left[x_{i}\right]$, the $\tau$-closed linear span of $\left(x_{i}\right)$, there is a unique sequence of scalars $\left(a_{i}\right)$ such that $x=\sum_{i=1}^{\infty} a_{i} x_{i}$. A basic sequence is unconditional if each expansion is u.c. A basic sequence in $X$ is of type $P$ if there is a $\tau$-neighborhood of $0, U$, in $X$ such that $x_{i} \notin U$ for each $i$ and $\left(\sum_{i=1}^{n} x_{i}\right)_{n=1}^{\infty}$ is $\tau$-bounded. It is known [9, p. 358] that if $\left(x_{i}\right)$ is an unconditional basic sequence of type $P$ in a Banach space $X$ then $\left[x_{i}\right]$ is isomorphic to $\left(c_{0}\right)$.

Proposition. Suppose $\left(x_{i}\right)$ is a basic sequence in a sequentially complete locally convex Hausdorff space $(X, \tau)$ and suppose $\sum_{i=1}^{\infty} x_{i}$ is $U B$ and that there is a $\tau$ neighborhood of $0, U$, in $X$ such that $x_{i} \notin U$ for each $i$. Then $\left(x_{i}\right)$ is an unconditional basic sequence of type $P$ and $\lambda\left(x_{i}\right)=\left\{\left(a_{i}\right): \sum_{i=1}^{\infty} a_{1} x_{i}\right.$ converges $\}=\left(c_{0}\right)$.

Proof. If $x=\sum_{i=1}^{\infty} a_{i} x_{i} \in\left[x_{i}\right]$ then, since $x_{i} \notin U$ for all $i$, we infer that $\left(a_{i}\right) \in\left(c_{0}\right)$. Since $\sum_{i=1}^{\infty} x_{i}$ is UB it follows from $\S 5$ (f) that $\left(a_{i}\right) \in \lambda\left(x_{i}\right)$ if $\left(a_{i}\right) \in\left(c_{0}\right)$. Thus $\lambda\left(x_{i}\right)$ $=\left(c_{0}\right)$. That $\left(x_{i}\right)$ is of type $P$ is clear. Since $\sum_{i=1}^{\infty} x_{i}$ is UB so is $\sum_{i=1}^{\infty} b_{i} x_{i}$ for $\left(b_{i}\right)$ a 0,1 sequence. Thus if $\left(a_{i}\right) \in \lambda\left(x_{i}\right)=\left(c_{0}\right)$, it follows again from $\S 5$ (f) that $\sum_{i=1}^{\infty} a_{i} b_{i} x_{i}$ $=\sum_{i=1}^{\infty} b_{i} a_{i} x_{i}$ converges (since $X$ is sequentially complete). Thus by $\S 4$ (b) $\sum_{i=1}^{\infty} a_{\imath} x_{i}$ converges unconditionally.

The above proposition is a generalization of [2, Lemma 3, p. 160].

THEOREM. In a sequentially complete locally convex Hausdorff space $(X, \tau)$ consider the following statements

(a) there is in $X$ a UB series which is not u.c.;

(b) there is in $X$ a UB series $\sum_{i=1}^{\infty} y_{n}$ and a $\tau$-neighborhood of $0, V$, such that $y_{n} \notin V$ for all $n$;

(c) there is in $X$ an (unconditional) basic sequence $\left(x_{i}\right)$ of type $P$ with $\lambda\left(x_{i}\right)=\left(c_{0}\right)$. Then (c) $\Rightarrow(\mathrm{a}) \Leftrightarrow(\mathrm{b})$. Moreover, if $X$ is a Frechet space then (a), (b) and (c) are mutually equivalent.

Proof. (c) $\Rightarrow$ (a). In view of the definition of type $P$ and $\$ 5$ (f) this implication is trivial. That (b) $\Rightarrow(\mathrm{a})$ is obvious. On the other hand if $\sum_{i=1}^{\infty} x_{i}$ is a UB series which is not u.c. then, for some permutation $p(n), \sum_{n=1}^{\infty} x_{p(n)}$ is not convergent. Thus there is a neighborhood $V$ of 0 in $E$ and an increasing sequence $\left(q_{n}\right)$ of positive integers such that

$$
y_{n}=\sum_{i=a_{n}+1}^{a_{n+1}} x_{p(i)} \notin V .
$$


It follows e.g. from $\$ 5$ (b) that $\sum_{n=1}^{\infty} y_{n}$ is UB. The last statement of the theorem has been observed in $[2,7.3$, p. 163$]$.

7. Some compact transformations determined by series. A linear transformation $T$ of one linear topological space to another is compact if it maps some neighborhood of 0 into a set whose closure is compact.

Lemma. If $S$ is a set, $X$ a linear topological space, $T_{n}$ and $T$ transformations from $S$ into $X$, then $T$ maps a subset $K$ of $S$ into a totally bounded subset $T(K)$ of $X$ provided

(a) $T_{n}(K)$ is totally bounded for each $n$; and,

(b) $\lim _{n} T_{n}(x)=T(x)$ uniformly for $x \in K$.

The proof of the lemma is straightforward and is omitted.

THEOREM. Let $X$ and $Y$ be locally convex Hausdorff spaces with $Y$ complete. Let $\left(f_{i}\right)$ be an equicontinuous sequence in $X^{*},\left(y_{i}\right)$ a sequence in $Y$ and $\left(\lambda_{i}\right)$ a sequence of scalars. For each $x \in X$ consider the formal series

$$
T(x)=\sum_{i=1}^{\infty} \lambda_{i} f_{i}(x) y_{i} .
$$

Then, if

(a) $\sum_{i=1}^{\infty} y_{i}$ is u.c. and $\left(\lambda_{i}\right) \in(m)$, or

(b) $\sum_{i=1}^{\infty} y_{i}$ is $U B$ and $\left(\lambda_{i}\right) \in\left(c_{0}\right)$, or

(c) $\left(y_{i}\right)$ is bounded and $\sum_{i=1}^{\infty}\left|\lambda_{i}\right|<+\infty$,

then $T$ defines a compact linear transformation from $X$ into $Y$.

Proof. Since $\left(f_{i}\right)$ is equicontinuous, $V=\left\{x \in X:\left|f_{i}(x)\right| \leqq 1\right\}$ is a neighborhood of 0 in $X$. For each $n$ let $T_{n}(x)=\sum_{i=1}^{n} \lambda_{i} f_{i}(x) y_{i}$ where $\left(\lambda_{i}\right)$ and $\left(y_{i}\right)$ satisfy any of (a), (b), or (c). Clearly $T_{n}(V)$ is totally bounded for each $n$. In case (a) observe that for $x \in X,\left(\lambda_{i} f_{i}(x)\right) \in(m)$ and so $T$ is well defined by $\S 4$ (a). Since $\left\{\left(\lambda_{i} f_{i}(x)\right): x \in V\right\}$ is a bounded subset of $(m)$ it follows from $\$ 4$ (e) that $T_{n}(x)$ converges to $T(x)$ uniformly on $V$. Since $Y$ is complete it follows from the lemma that $T$ is compact.

In case (b) if $\left(\lambda_{i}\right) \in\left(c_{0}\right)$ then by $\S 5$ (f) $\sum_{i=1}^{\infty} \lambda_{i} y_{i}$ is u.c. Now apply (a) with the scalar sequence consisting of all 1's.

Again in case (c) $\sum_{i=1}^{\infty} \lambda_{i} y_{i}$ is u.c. and the theorem follows from (a).

Let us observe that the compact operators determined by (c) above are precisely the nuclear operators. For if $\left(y_{i}\right)$ is bounded and $B$ is the closed circled convex hull of $\left(y_{i}\right)$ then, since $Y$ is complete, $B$ is complete. Thus the normed linear space $Y_{B}=\bigcup_{n=1}^{\infty} n B$ with norm the gauge of $B$ is a Banach space. That $T$ is nuclear now follows from $[8$, p. 99].

In a manner analogous to the above, one can prove the following known result: Let $H_{1}$ and $H_{2}$ be Hilbert spaces and $\left(\varphi_{i}\right)$ and $\left(\psi_{i}\right)$ orthonormal sequences in $H_{1}$ and 
$H_{2}$ respectively. If $\left(\lambda_{i}\right) \in\left(c_{0}\right)$ then $T(x)=\sum_{i=1}^{\infty} \lambda_{i}\left(x, \varphi_{i}\right) \psi_{i}, x \in H_{1}$, defines a compact map from $H_{1}$ into $H_{2}$. In fact every compact Hermitian operator $T$ from a Hilbert space into itself is of the above form (see e.g. [4]).

8. A stability theorem for bases in Banach spaces. Let us recall the classical Paley-Wiener stability theorem: Let $\left(x_{i}\right)$ and $\left(y_{i}\right)$ be sequences in a Banach space $X$ and let $0<\lambda<1$. Assume that $\left(x_{i}\right)$ and $\left(y_{i}\right)$ satisfy

$$
\left\|\sum_{i=1}^{p} t_{i}\left(x_{i}-y_{i}\right)\right\| \leqq \lambda\left\|\sum_{i=1}^{p} t_{i} x_{i}\right\|
$$

for arbitrary scalars $t_{1}, \ldots, t_{p}$ and arbitrary positive integer $p$. Then

(a) if $\left[x_{i}\right]=X$ then $\left[y_{i}\right]=X$;

(b) if $\left(x_{i}\right)$ is a basic sequence so is $\left(y_{i}\right)$ and whenever $\sum_{i=1}^{\infty} b_{i} x_{i}$ converges,

$$
\left\|\sum_{i=1}^{\infty} b_{i} x_{i}\right\| \leqq \frac{1}{1-\lambda}\left\|\sum_{i=1}^{\infty} b_{i} y_{i}\right\| ;
$$

(c) if $\left(x_{i}\right)$ is basic there is a linear homeomorphism $T$ of $\left[x_{i}\right]$ onto $\left[y_{i}\right]$ such that $T\left(x_{i}\right)=y_{i}$ for each $i$ (see [1] and [7]).

A biorthogonal system $\left(x_{i}, f_{i}\right)$ in a Banach space $X$ is a pair of sequences, $\left(x_{i}\right) \subset X$, $\left(f_{i}\right) \subset X^{*}$ such that $f_{i}\left(x_{j}\right)=\delta_{i j}$.

THEOREM. Let $\left(x_{i}, f_{i}\right)$ be a biorthogonal system in a Banach space $X$ with sup $_{n}\left\|f_{n}\right\|$ $=M<+\infty$. Let $0<\lambda<1$ and suppose $\left(y_{i}\right)$ is a sequence in $X$ such that

$$
\sup \left\{\left\|\sum_{i \in \sigma}\left(x_{i}-y_{i}\right)\right\|: \sigma \in \Phi\right\} \leqq \frac{\lambda}{4 M} .
$$

Then $\left(x_{i}\right)$ and $\left(y_{i}\right)$ satisfy (P-W) and so the conclusions of the Paley-Wiener theorem hold. If $X$ is a real Banach space the number 4 above may be replaced by 2 .

Proof. Let $\left(t_{i}\right)_{i=1}^{p}$ be an arbitrary finite set of scalars and let $x=\sum_{i=1}^{p} t_{i} x_{i}$. Then $f_{i}(x)=t_{i}$ if $i \leqq p$ and from (I) we obtain

$$
\begin{aligned}
\left\|\sum_{i=1}^{p} t_{i}\left(x_{i}-y_{i}\right)\right\| & \leqq \frac{\lambda}{M}\left(\sup _{i \leqq p}\left|t_{i}\right|\right)=\frac{\lambda}{M}\left(\sup _{i \leqq p}\left|f_{i}(x)\right|\right) \\
& \leqq \lambda\left\|\sum_{i=1}^{p} t_{i} x_{i}\right\| .
\end{aligned}
$$

In [5] Krein, Milman and Rutman proved that if $\left(x_{i}\right)$ is a basis for $X$ and $f_{i}(x)$ $=f_{i}\left(\sum_{j=1}^{\infty} a_{j} x_{j}\right)=a_{i}$ then a sequence $\left(y_{i}\right)$ in $X$ satisfying

$$
\sum_{i=1}^{\infty}\left\|f_{i}\right\|\left\|x_{i}-y_{i}\right\|=\lambda<1
$$

is also a basis for $X$.

In [10] Veic proved that if $\left(x_{i}\right)$ is a basis for $X$ with $0<\inf _{n}\left\|x_{n}\right\| \leqq \sup _{n}\left\|x_{n}\right\|<+\infty$ 
then an $\omega$-independent sequence $\left(y_{i}\right)\left(\sum_{i=1}^{\infty} a_{i} y_{i}=0 \Rightarrow a_{i}=0\right.$ for each $\left.i\right)$ satisfying

$$
\sum_{i=1}^{\infty} x_{i}-y_{i} \text { is u.c. }
$$

is also a basis for $X$. (Weill [11] has demonstrated the validity of this result in complete barrelled spaces.)

Let us observe that in weakly sequentially complete Banach spaces the Veic stability theorem is more general than our result for we place a specific bound on $\left\|\sum_{i=1}^{\infty} x_{i}-y_{i}\right\|$ in such spaces.

The statement in [10] that the theorem using (V) is more general than the (K-M-R) theorem is not, strictly speaking, valid. Observe that using (K-M-R) there is no boundedness requirement placed on $\left(x_{i}\right)$ nor is $\omega$-independence of $\left(y_{i}\right)$ hypothesized (however, that $\left(y_{i}\right)$ must be $\omega$-independent easily follows from the other hypotheses).

In the following examples it becomes evident that sequences may satisfy one of (K-M-R), (V) or (M-R) without satisfying any other. Before proceeding to the examples let us observe that all three theorems are strong enough to prove the following fundamental fact: In a Banach space with a basis, a basis may be chosen from any dense set, a fact first observed in [5].

EXAmple 1. Sequences satisfying (K-M-R) but not (V) or (M-R). Let $X=\left(c_{0}\right)$ and let $x_{n}=\left(n \delta_{i n}\right)_{i=1}^{\infty}$. Then $f_{n}=\left((1 / n) \delta_{i n}\right)_{i=1}^{\infty}$. Let $y_{n}$ be the element of $\left(c_{0}\right)$ defined by $y_{n}(i)=1 / 2 n$ for $i=1,2, \ldots, n-1$ and $y_{n}(i)=n-1 / 2 n$ for $i=n$ and $y_{n}(i)=0$ for $i>n$. Then $\left\|x_{n}-y_{n}\right\|=1 / 2 n$ for $n \geqq 1$ and so

$$
\sum_{n=1}^{\infty}\left\|f_{n}\right\|\left\|x_{n}-y_{n}\right\|=\sum_{n=1}^{\infty} \frac{1}{2 n^{2}}=\frac{\pi^{2}}{12}<1
$$

and so (K-M-R) holds.

Let $h_{n}=\sum_{i=1}^{n}(1 / i)$ and let $a_{n 1}$ denote the first coordinate of $\sum_{i=1}^{n}\left(y_{i}-x_{i}\right)$. An easy calculation shows that $a_{n 1}=\frac{1}{2}\left(h_{n}-2\right)$ whence $\sup _{n}\left\|\sum_{i=1}^{n} x_{i}-y_{i}\right\| \geqq \sup _{n}\left|a_{n 1}\right|$ $=+\infty$ and so (M-R) does not hold. Clearly (V) does not hold.

EXAMPLE 2. Sequences satisfying (M-R) but not (K-M-R) or (V).

Again let $X=\left(c_{0}\right)$ and let $\left(x_{n}\right)$ denote the unit vector basis of $\left(c_{0}\right)$. For $0<\lambda<1$ let $y_{n}=(1-\lambda / 4) x_{n}$. Then $\sup _{\sigma}\left\|\sum_{i \in \sigma} x_{i}-y_{i}\right\|=\lambda / 4$ and (M-R) holds. However $\sum_{i=1}^{\infty} x_{i}-y_{i}=\sum_{i=1}^{\infty}(\lambda / 4) x_{i}$ is not u.c. Since, if $\left(f_{n}\right)$ is the sequence of functionals associated with $\left(x_{n}\right),\left\|f_{n}\right\|=1$, we see that neither $(\mathrm{V})$ nor $(\mathrm{K}-\mathrm{M}-\mathrm{R})$ hold.

EXAMPLE 3. Sequences satisfying (V) but not (M-R) or (K-M-R).

Again let $X=\left(c_{0}\right)$ and $\left(x_{n}\right)$ be the unit vector basis of $\left(c_{0}\right)$. Let $y_{1}=2 x_{1}$ and for $i \geqq 2$ let $y_{i}=(i-1) x_{i} / i$. Then $x_{i}-y_{i}=x_{i} / i, i \geqq 2$ and $x_{1}-y_{1}=-x_{1}$. Clearly $\sum_{i=1}^{\infty} x_{i}-y_{i}$ is u.c. and $\left\|\sum_{i=1}^{\infty} x_{i}-y_{i}\right\|=1$. Thus (V) is satisfied but not (M-R) or (K-M-R).

The next two examples illustrate the scope of our stability theorem. In both examples $X=\left(c_{0}\right)$ and $\left(x_{i}\right)$ is the unit vector basis of $\left(c_{0}\right)$.

EXAMPLE 4. Let $\varepsilon>0$ be given. Let $y_{1}=x_{1}$ and $y_{n}=(-1)^{n+1} \varepsilon x_{1}+x_{n}$ for $n \geqq 2$. 
Then $x_{n}-y_{n}=(-1)^{n+2} \varepsilon x_{1}$ for $n \geqq 2$ while $x_{1}-y_{1}=0$. Thus $\sup _{n}\left\|\sum_{i=1}^{n} x_{1}-y_{1}\right\|=\varepsilon$. However $\left(y_{n}\right)$ is not a basic sequence in $\left(c_{0}\right)$. To see this suppose there is a $g \in X^{*}$ such that $g\left(y_{1}\right)=1$ and $g\left(y_{n}\right)=0, n \geqq 2$ (such exists if $\left(y_{n}\right)$ is a basic sequence). Observe that $\varepsilon y_{1}-y_{2 K-1}=x_{2 K-1}$ and $\lim _{K} g\left(x_{2 K-1}\right)=0$. Thus

$$
\varepsilon=\lim g\left(\varepsilon y_{1}-y_{2 K-1}\right)=\lim _{n} g\left(x_{2 K-1}\right)=0 .
$$

Thus we see that our stability theorem is false if unordered boundedness is replaced by ordered boundedness.

The next example shows that, in a certain sense, our stability theorem is the best possible. For, the example shows that even $\left(x_{i}\right)$ being a basis, $\sup _{i}\left\|f_{i}\right\|<+\infty,\left(y_{i}\right)$ $\omega$-independent and $\sup _{\sigma}\left\|\sum_{i \in \sigma} x_{i}-y_{i}\right\|<+\infty$ need not imply that $\left(y_{i}\right)$ is a basic sequence.

EXAMPLE 5. Again with $\left(x_{i}\right)$ the unit vector basis of $\left(c_{0}\right)$ let $y_{1}=x_{1}$ and $y_{n}=x_{n-1}$ $-x_{n}$ for $n \geqq 2$. Then $\left(y_{n}\right)$ has the asserted properties. For, if $\sum_{i=1}^{\infty} a_{i} y_{i}=0$ then, by applying $f_{j}$ (the coefficient functionals associated with $\left(x_{i}\right)$ ) we obtain $a_{1}+a_{2}=0$, $a_{n}=a_{n-1}, n \geqq 3$. Since $\left\|\sum_{i=p}^{q} y_{i}\right\|=1$ if $q>p \geqq 2$ and $\sum_{i=2}^{\infty} a_{i} y_{i}$ converges we see that $a_{i}=0$ for all $i$ and so $\left(y_{i}\right)$ is $\omega$-independent.

Clearly $\sup _{\sigma}\left\|\sum_{i \in \sigma} \dot{x}_{i}-y_{i}\right\|=2$.

Finally observe that $y_{1}-\sum_{i=2}^{n} y_{i}=x_{n}$ and as in Example 4 it follows that $\left(y_{i}\right)$ cannot be a basic sequence.

As a final remark let us observe that it follows from [2, Lemma 3, p. 160] (see also $\S 6$, Proposition) that if $\left(x_{i}\right)$ is a basic sequence with uniformly bounded coefficient functionals $\left(f_{n}\right)$ and if $\left(y_{i}\right)$ is a sequence such that both $\sum_{i=1}^{\infty} x_{i}-y_{i}$ and $\sum_{i=1}^{\infty} y_{i}$ are UB then $\left(x_{i}\right)$ is an unconditional basis of type $P$ and so $\left[x_{i}\right]$ is isomorphic

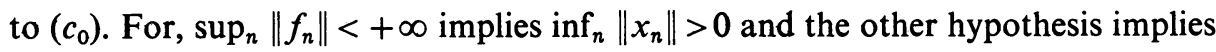
$\sum_{i=1}^{\infty} x_{i}$ is UB.

In particular, our stability theorem seems most interesting whenever

$$
\sup _{\sigma}\left\|\sum_{i \in \sigma} y_{i}\right\|=+\infty
$$

\section{BIBLIOGRAPHY}

1. M. G. Arsove, The Paley-Wiener theorem in metric linear spaces, Pacific J. Math. 10 (1960), 365-379.

2. C. Bessaga and A. Pelczynski, On bases and unconditional convergence of series in Banach spaces, Studia Math. 17 (1958), 151-164.

3. M. M. Day, Normed linear spaces, Ergebnisse der Mathematik, Springer-Verlag, Berlin, 1958.

4. J. Dixmier, Les fonctionnelles linéaires sur l'ensemble des opérateurs bornés d'un espace de Hilbert, Ann. of Math. 51 (1950), 387-408.

5. M. Krein, D. Milman and M. Rutman, On a property of a basis in a Banach space, Kharkov. Zap. Mat. Obšč. (4) 16 (1940), 182.

6. C. W. McArthur, A convergence criterion with applications to series in locally convex spaces, Duke Math. J. 34 (1967), 193-200. 
7. J. R. Retherford, Basic sequences and the Paley-Wiener criterion, Pacific J. Math. 14 (1964), 1019-1027.

8. H. H. Schaefer, Topological vector spaces, Macmillan, New York, 1966.

9. I. Singer, Bases, basic sequences and reflexivity of Banach spaces, Studia Math. 21 (1962), 351-369.

10. B. E. Veic, Some stability properties of bases, Soviet Math. Dokl. 5 (1964), 1141-1144.

11. L. Weill, Stability of bases in complete barrelled spaces, Proc. Amer. Math. Soc. 18 (1967), 1045-1050.

Florida State University,

TAllahassee, Florida

Louisiana State UNIVERSity,

Baton Rouge, Louisiana 